Chronic Obstructive Pulmonary Diseases: Journal of the COPD Foundation

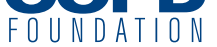

\author{
Images in COPD Case Report
}

\title{
Thoracic Computed Tomography Features of Coronavirus Disease 2019 Patients with Emphysema
}

\author{
Stephanie Tittaferrante, $\mathrm{MD}^{1^{*}}$ Rohit Gupta, $\mathrm{MD}^{2^{*}}$ Victor Kim, $\mathrm{MD}^{2}$ on behalf of the Temple University \\ COVID-19 Research Group
}

\begin{abstract}
Abbreviations: computed tomography, CT; coronavirus disease 2019, CovID-19; severe acute respiratory syndrome coronavirus-2, SARS-CoV-2; chronic obstructive pulmonary disease, COPD; polymerase chain reaction, PCR; intravenous immune globulin, IVIG Date of Acceptance: June 9, 2020

Citation: Tittaferrante S, Gupta R, Kim V, Temple University Research Group. Thoracic computed tomography features of coronavirus disease 2019 patients with emphysema. Chronic Obstr Pulm Dis. 2020;7(3):290-296. doi: https://doi.org/10.15326/jcopdf.7.3.2020.0166
\end{abstract}

1 Department of Internal Medicine, Temple University Health System, Philadelphia, Pennsylvania

2 Department of Thoracic Medicine and Surgery, Temple University Health System, Philadelphia, Pennsylvania

* These authors contributed equally as first authors. A list of individuals in the Temple University COVID-19 Research Group is provided in the online data supplement.

\section{Address correspondence to:}

Stephanie Tittaferrante, MD

Department of Internal Medicine and Surgery

Temple University Health System

Philadelphia, Pennsylvania

Email: stephanie.tittaferrante@tuhs.temple.edu

\section{Keywords:}

coronavirus disease 2019; COVID-19; emphysema; computed tomography imaging: CT scans

\section{Introduction}

This case series presents the thoracic computed tomography (CT) findings of coronavirus disease of 2019 (COVID-19) infection in 8 patients with a background of emphysema. While the predominant imaging findings were bilateral groundglass opacities with areas of consolidation as typically seen with COVID-19, patterns appear to be variable. The interplay between the underlying chronic lung disease and the acute viral illness in generating specific chest imaging findings needs to be better defined by larger studies.

COVID-19 is caused by severe acute respiratory syndrome coronavirus-2 (SARS-CoV-2) and can lead to mild or severe respiratory illness. ${ }^{1}$ Patients with chronic obstructive pulmonary disease (COPD) are at increased risk of developing severe infection and have higher mortality rates. ${ }^{2,3}$ Chest $\mathrm{X}$-ray, unenhanced chest $\mathrm{CT}$ and reverse transcription polymerase chain reaction (PCR) have been used to aid diagnosis. Chest $\mathrm{CT}$ has been shown to be more rapid and more sensitive than PCR for the diagnosis of COVID-19, and these are important test characteristics in a pandemic setting. ${ }^{4,5}$ Chest $\mathrm{CT}$ also provides an estimate of disease burden and might be useful for management decisions. The most specific CT findings of COVID-19 pneumonia include bilateral peripheral groundglass opacities with or without consolidation and multifocal, rounded ground glass opacities with or without consolidation. ${ }^{6}$ We present a series of patients with a background of emphysema who were diagnosed with COVID-19 pneumonia based on clinical features and nasopharyngeal PCR.

\section{Case Descriptions and Imaging Case 1}

Case 1 is a 49-year-old woman with severe COPD and chronic hypoxic respiratory failure on 2L oxygen, tracheal stenosis, systolic heart failure, history of pulmonary embolism, and left ventricular thrombus on anticoagulation who presented with 4 days of progressively worsening dyspnea on exertion, cough, and orthopnea. She was found to have acute on chronic hypoxic respiratory failure due to COVID-19 pneumonia, needing up to 5L oxygen. She was treated 
with antibiotics and steroids and was weaned back down to her baseline oxygen need prior to discharge. (Figure $1 \mathrm{a}$ and $\mathrm{b}$ ).

\section{Case 2}

Case 2 is a 76-year-old man with severe COPD and chronic hypoxic respiratory failure on 3L oxygen, history of bilateral lung volume reduction surgery who presented with 3 weeks of dyspnea and productive cough. He was found to have acute on chronic hypoxic respiratory failure due to COVID-19 pneumonia needing up to 5L of oxygen. He was treated with a 5 -day course of ceftriaxone and azithromycin and a 15-day course of steroids. He was weaned back down to baseline oxygen need at time of discharge. (Figure 2)

\section{Case 3}

Case 3 is a 63 -year-old woman with COPD, morbid obesity, diabetes and hypertension who presented with 5 days of dyspnea, headache, vomiting, and chills. She was found to have acute hypoxic respiratory failure

\section{Figure 1. Baseline Chest Computed Tomography (a) and Chest Computed Tomography at Time of Admission (b)}
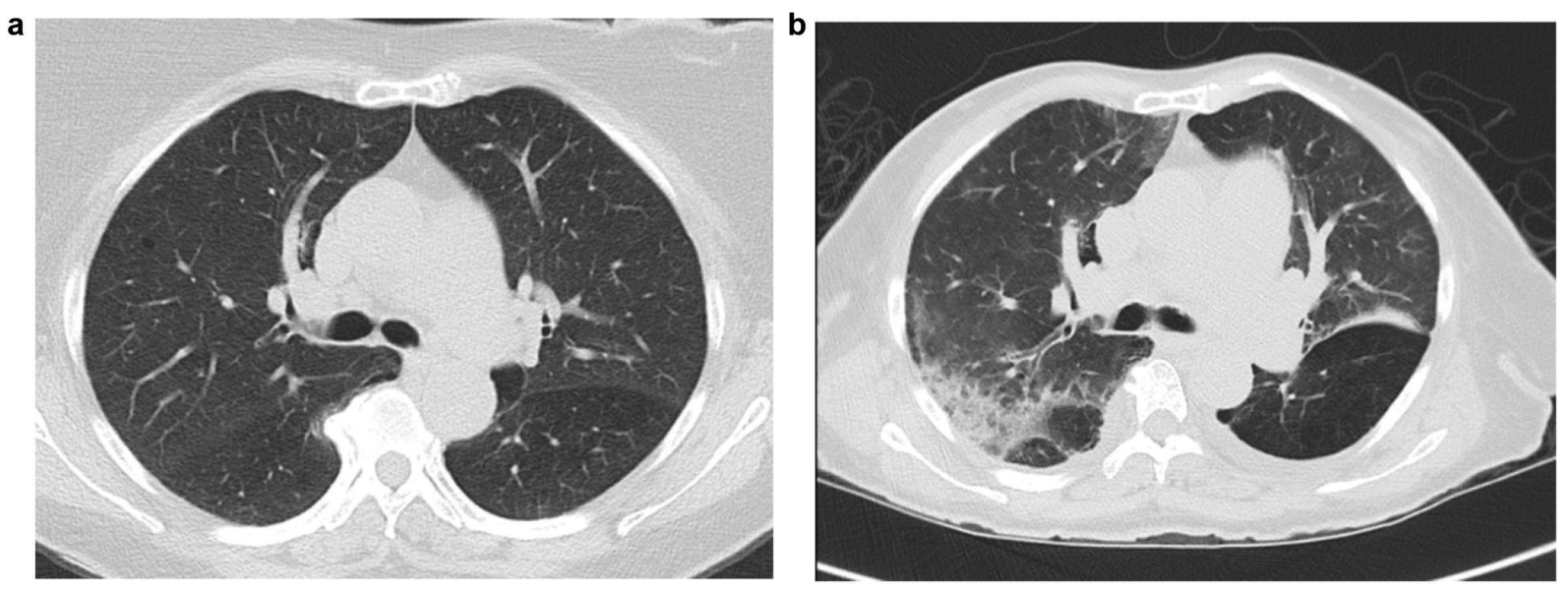

Baseline chest CT (08/2015) showing mild centrilobular emphysema (a) and chest CT from time of admission with COVID-19 showing diffuse groundglass opacities and consolidation in the right lung (b).

$\mathrm{CT}=$ computed tomography

\section{Figure 2. Chest Computed Tomography Showing Severe Emphysema}
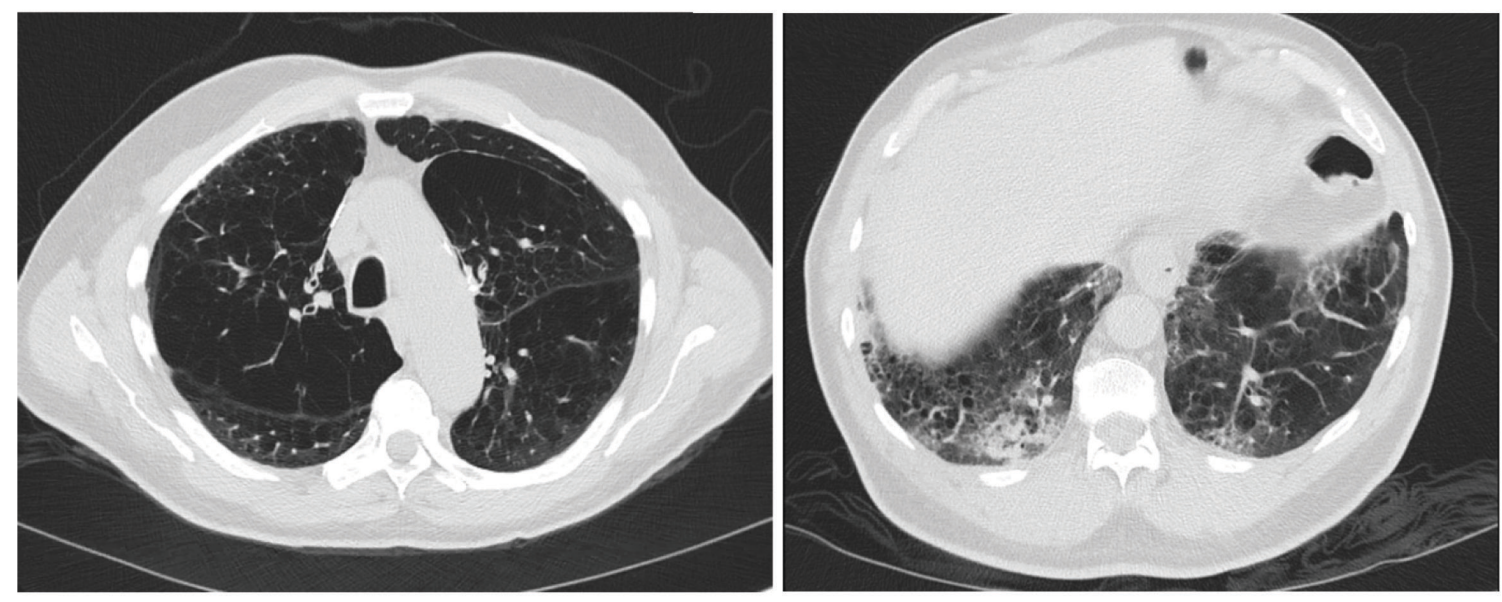

Chest CT showing severe emphysema at the apices and scattered groundglass opacities with consolidative opacities at the bases.

$\mathrm{CT}=$ computed tomography 
due to COVID-19 pneumonia requiring mechanical ventilation. She was treated with antibiotics, hydroxychloroquine, and was enrolled in a clinical trial. Her hospital course was complicated by ventilatorydependent respiratory failure requiring tracheostomy. She was discharged to a long-term acute care facility. (Figure 3)

\section{Case 4}

Case 4 is a 55-year-old woman with hypertension who presented with 1 week of nocturnal cough associated with dyspnea and hemoptysis without constitutional symptoms. She was found to have acute hypoxic respiratory failure due to COVID-19 pneumonia requiring up to $4 \mathrm{~L}$ oxygen. She was also found to have small cell carcinoma causing compression of her right mainstem bronchus which required stent placement. She was treated with ceftriaxone and azithromycin and was discharged with a plan to start outpatient chemotherapy. (Figure 4)

\section{Case 5}

Case 5 is a 78-year-old man with COPD, chronic lymphocytic leukemia on acalabrutinib and hypogammaglobulinemia on intravenous immune globulin (IVIG) who presented with 1 week of dyspnea, cough, malaise, and diarrhea. He was found to have acute hypoxic respiratory failure due to COVID-19 pneumonia requiring intubation. He was treated with azithromycin, ceftriaxone, steroids, IVIG, and was

\section{Figure 3. Chest Computed Tomography Showing Emphysema and Bilateral Peripheral Groundglass Opacities with Some Areas of Consolidation}
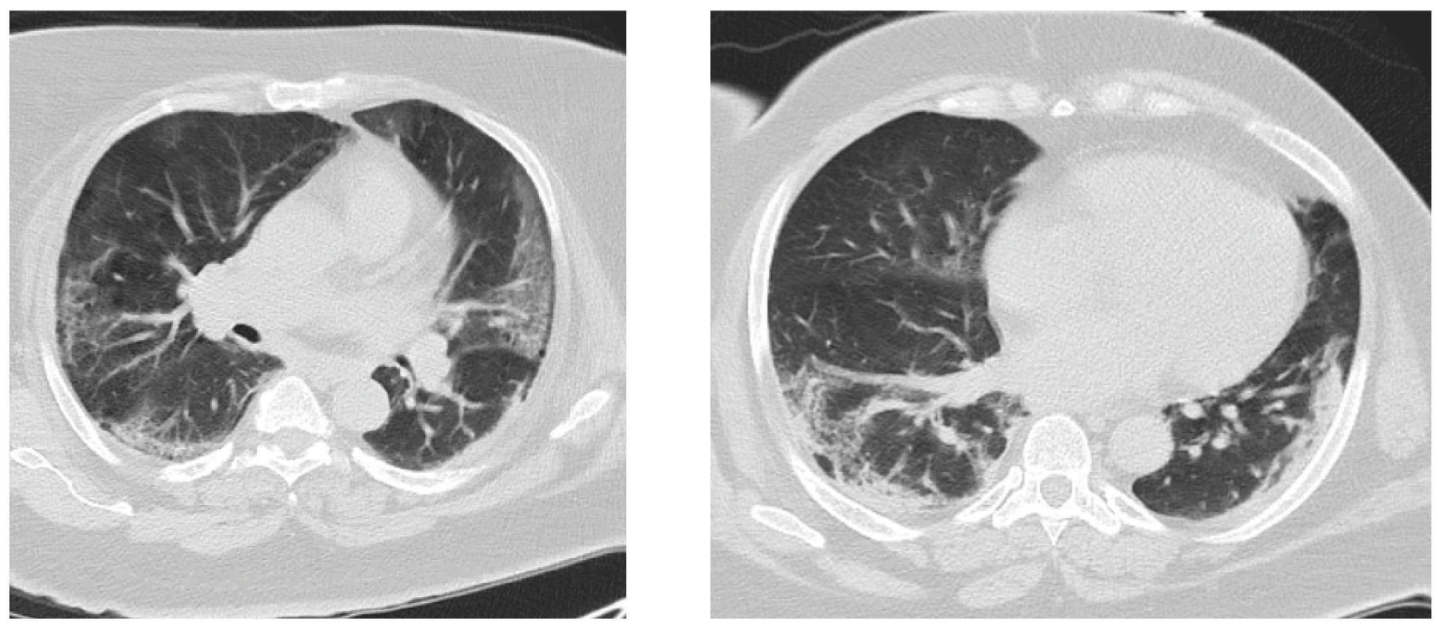

\section{Figure 4. Chest Computed Tomography Showing Emphysema and Diffuse Groundglass Opacities}
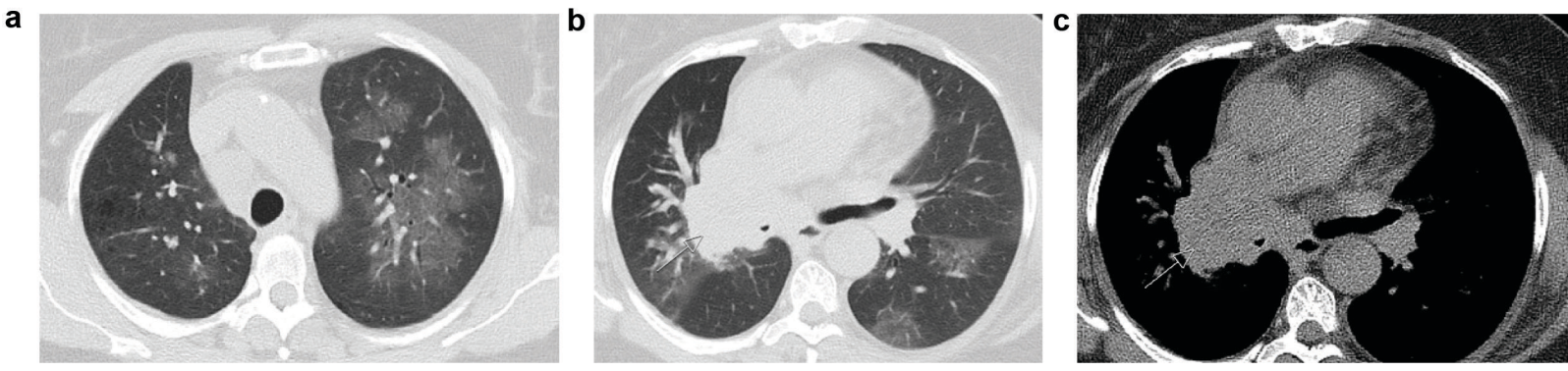

Chest CT showing emphysema and diffuse groundglass opacities which are more pronounced in the left lung. The arrows in $4 \mathrm{~b}$ and $4 \mathrm{c}$ point to a right hilar mass causing compression of the right mainstem bronchus. 
enrolled in a clinical trial. His hospital course was complicated by pulmonary embolism involving the distal segmental arteries of the right upper lobe and left lower lobe. The patient was ultimately transitioned to comfort care where he died. (Figure 5)

\section{Case 6}

Case 6 is a 42-year-old man with COPD, systolic heart failure, severe aortic stenosis and hypertension who presented with 2 months of progressively worsening dyspnea, productive cough, and diarrhea and was found to have acute hypoxic and hypercapnic respiratory failure due to COVID-19 pneumonia requiring noninvasive ventilation. He was treated with steroids, azithromycin, ceftriaxone, diuresis, and was discharged on 3L of oxygen. (Figure 6)

\section{Case 7}

Case 7 is a 76-year-old woman with COPD, hypertension and history of a cerebrovascular accident who presented from her nursing home for respiratory distress. She was found to have acute hypoxic respiratory failure due to COVID-19 pneumonia initially requiring non-rebreather. She was treated with steroids, ceftriaxone, azithromycin, IVIG, and anakinra. She was weaned to room air prior to discharge. (Figure 7)

\section{Figure 5. Chest Computed Tomography Showing Emphysema and Groundglass Opacities}
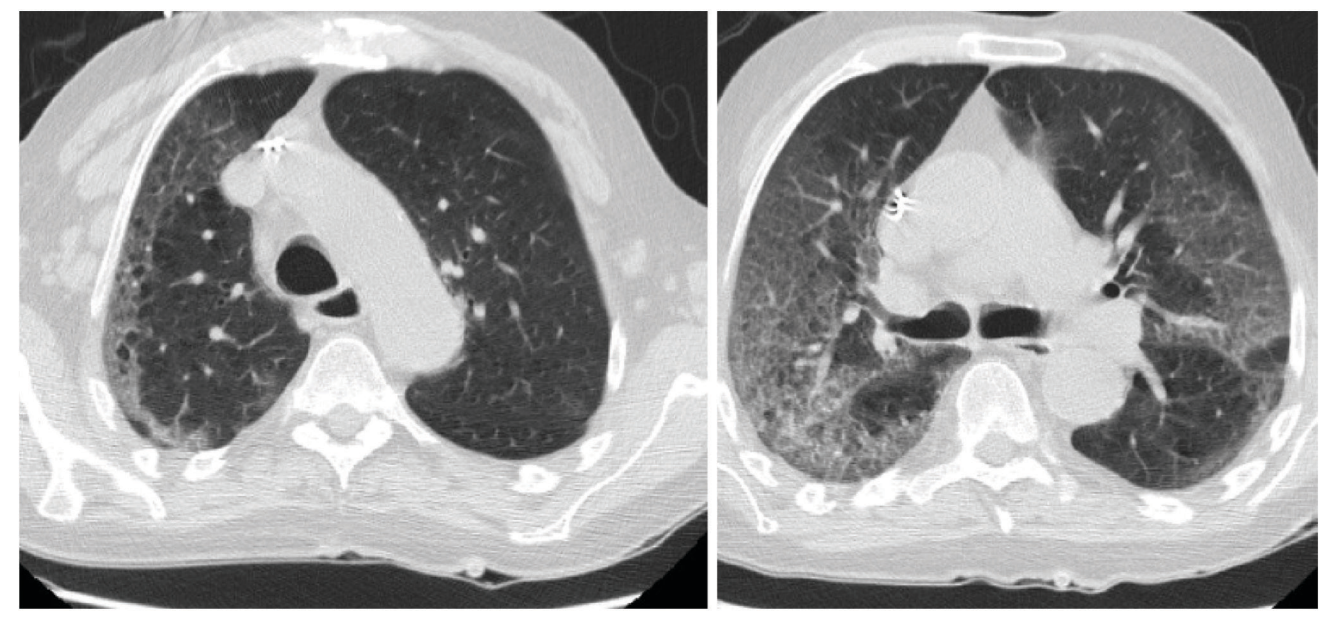

Chest CT showing emphysema and groundglass opacities predominantly located peripherally at the apices but more diffuse towards the bases.

\section{Figure 6. Chest Computed Tomography Showing Emphysema and Extensive Multifocal Groundglass Opacities}
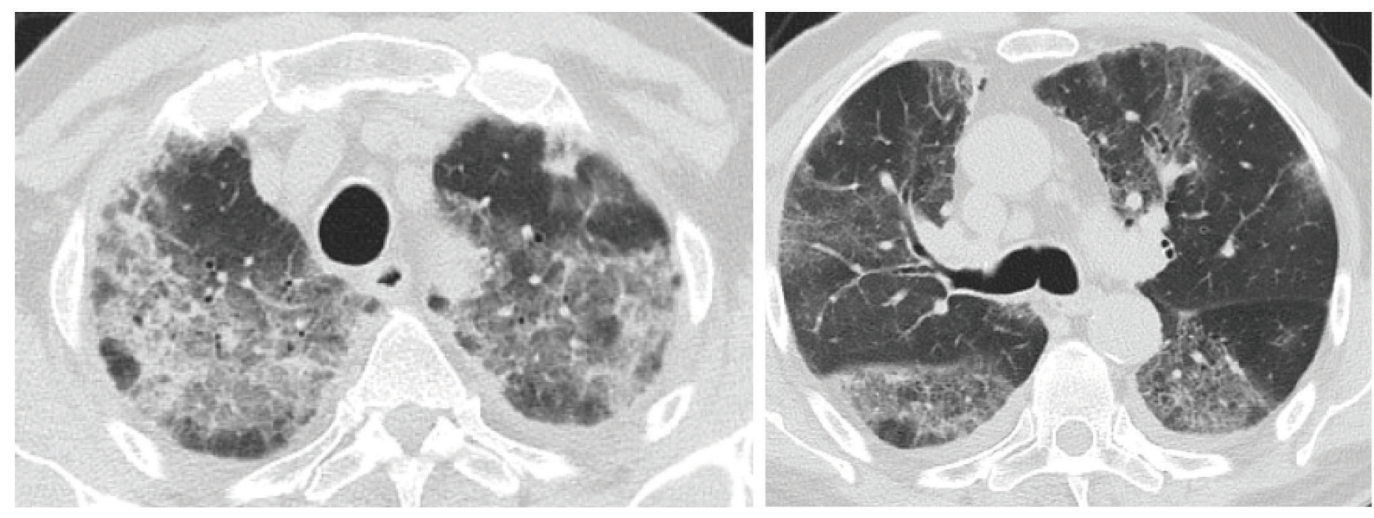


\section{Case 8}

Case 8 is a 53-year-old woman with COPD and hypertension who presented with several days of abdominal pain and dyspnea. She was found to have acute hypoxic respiratory failure due to COVID-19 pneumonia requiring mechanical ventilation. She was also found to have a small bowel perforation due to bowel ischemia from microvascular thrombosis and required ileal resection with end ileostomy. She was treated with azithromycin, steroids, convalescent plasma, IVIG, and anakinra. Her hospital course was complicated by acute respiratory distress syndrome and septic shock due to pseudomonal pneumonia requiring rescue therapies for severe hypoxia. She died despite maximal supportive measures. (Figure 8)

\section{Figure 7. Chest Computed Tomography Showing Emphysema and Peripheral Groundglass Opacities Predominantly at Left Base}
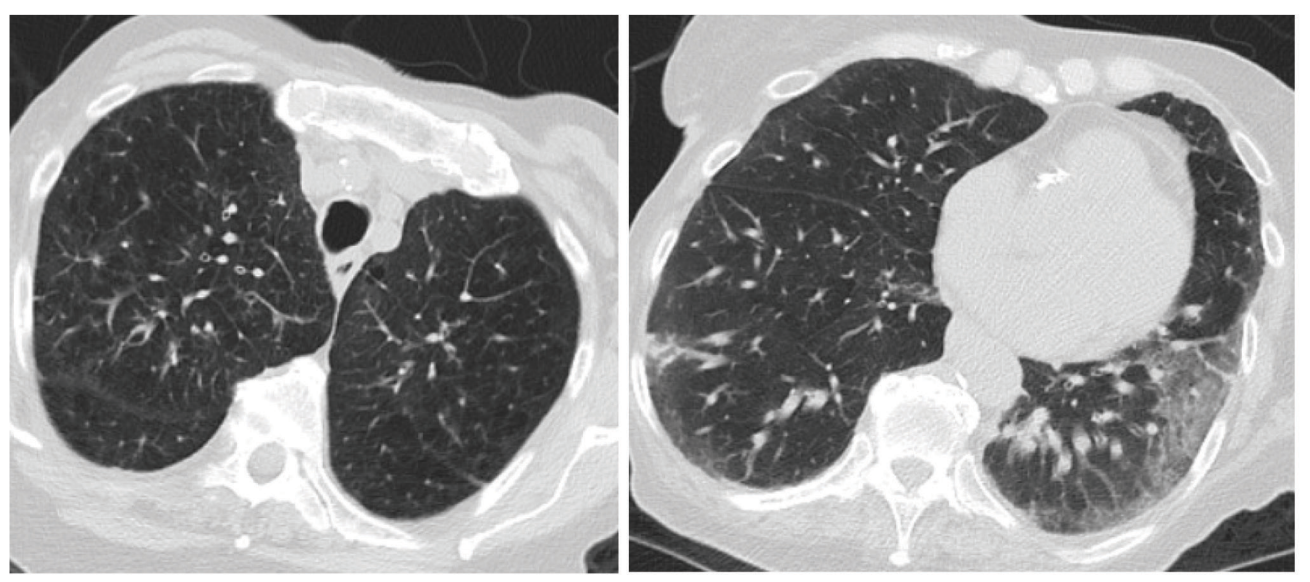

Figure 8. Chest Computed Tomography Showing Emphysema and Biateral Peripheral "Rim-like" Groundglass Opacities with Areas of Consolidation at the Bases
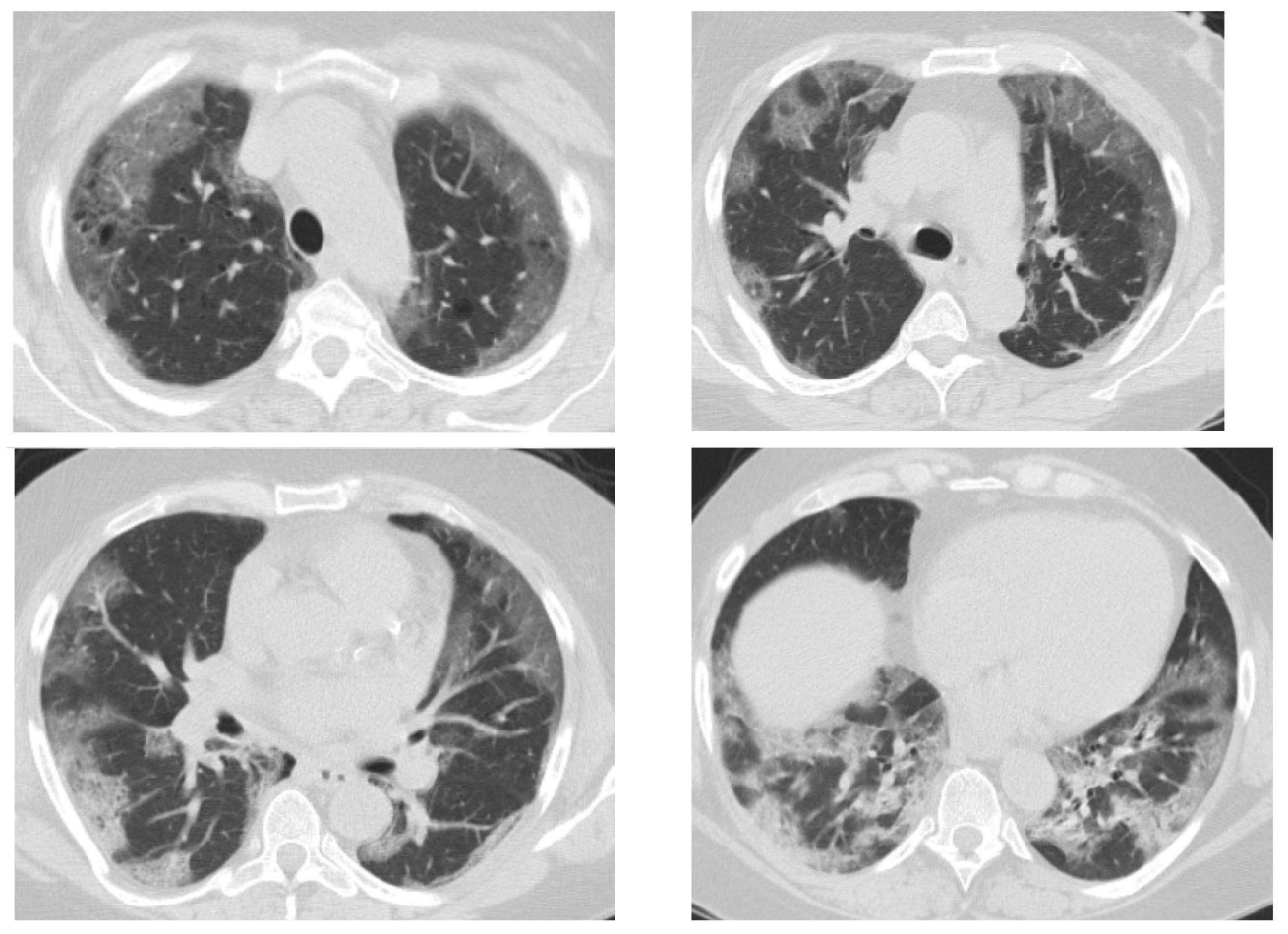


\section{Discussion}

COVID-19 can cause severe respiratory illness in patients with COPD, and chest CTs have been shown to be a sensitive test for the diagnosis of COVID-19 pneumonia. 4 Typically, COVID-19 causes bilateral, peripheral groundglass opacities with or without consolidation and multifocal, rounded groundglass opacities with or without consolidation on chest CT. ${ }^{5}$ This case series describes the imaging findings in patients with emphysema who were confirmed to have COVID-19. As is typically seen with COVID-19, many patients had bilateral groundglass opacities with areas of consolidation; however, the pattern was variable. Most patients had more pronounced disease in the lung bases. None of the patients had rounded groundglass opacities. While some imaging features are more typical of COVID-19 pneumonia, imaging characteristics can differ in patients with underlying lung disease. These may depend on the extent and severity of underlying lung disease as well as the acute viral illness. 


\section{References}

1. World Health Organization. Coronavirus disease (COVID-19) pandemic. Updated June 2020. Accessed 19 May 2020. https:// www.who.int/emergencies/diseases/novel-coronavirus-2019

2. Zhao Q, Meng M, Kumar R, et al. The impact of COPD and smoking history on the severity of COVID-19: a systemic review and meta-analysis. J Med Virol. 2020 Apr 15.

doi: https://doi.org/10.1002/jmv.25889

3. Alqahtani JS, Oyelade T, Aldhahir AM, et al. Prevalence, severity and mortality associated with COPD and smoking in patients with COVID-19: a rapid systematic review and meta-analysis. Plos One. 2020 May 11;15(5):e0233147.

doi: https://doi.org/10.1371/journal.pone.0233147

4. Fang Y, Zhang H, Xie J, et al. Sensitivity of chest CT for COVID-19: comparison to RT-PCR. Radiology. 2020 Feb 19:200432. doi: https://doi.org/10.1148/radiol.2020200432

5. Li Y, Xia L. Coronavirus disease 2019 (COVID-19): role of chest $\mathrm{CT}$ in diagnosis and management. AJR Am J Roentgenol. 2020 Mar 4:1-7. doi: https://doi.org/10.2214/AJR.20.22954

6. Simpson S, Kay FU, Abbara S, et al. Radiological Society of North America expert consensus statement on reporting chest CT findings related to COVID-19. Endorsed by the Society of Thoracic Radiology, the American College of Radiology, and RSNA. Radiol Cardiothorac Imaging. 2020 Mar 25;2(2):e200152. doi: https://doi.org/10.1148/ryct.2020200152 